\title{
„Meine Aufgabe ist, einen Bericht zu schreiben“: „Die sanften Kontrolleure“ und ihre Dokumente
}

\author{
Sylvia Kühne $\cdot$ Christina Schlepper
}

Online publiziert: 21. Januar 2020

(C) Der/die Autor(en) 2019

Zusammenfassung Dieser Beitrag untersucht - aus der Perspektive der institutionellen Ethnographie und unter Rückgriff auf Datenmaterial der vergleichenden Replikationsstudie „Die sanften Kontrolleure“ - wie Sozialarbeiter_innen im Handlungsfeld Jugendgerichtshilfe Dokumente in Gesprächen mit ihren Adressat_innen zum Einsatz bringen. Es zeigt sich, dass Dokumente Jugendgerichtshelfer_innen einerseits dazu dienen, Konflikte zu vermeiden und die Hilfeabsicht die Interaktion bestimmen zu lassen. Andererseits werden auch Funktionen offenbar, die in Strategien der Wahrheitsfindung eingebettet sind und nicht auf Hilfe für die Adressat_innen, sondern auf die Erfüllung des justiziellen Handlungsauftrags zielen. In variierendem Ausmaß fungiert die Akte als Verbündete in der Interaktion und erweitert das sozialarbeiterische Handlungsrepertoire um Möglichkeiten, sich als Helfer_in darzustellen - jedoch nicht nur der Adressat_innen, sondern vor allem des Gerichts. So deuten die Ergebnisse, erstens, darauf hin, dass der Einbezug von Dokumenten in die Interaktion die Gefahr in sich birgt, dass Sozialarbeiter_innen ihre Handlungspraxis standardisieren und dabei eine juristische Handlungslogik antizipieren. Analog dazu weist, zweitens, auch ein diachroner Vergleich von Bezugnahmen auf Dokumente in den Gesprächen zwischen Sozialarbeiter_innen und ihren Adressat_innen auf eine Verschiebung der Selbstpositionierungen von Sozialarbeiter_innen in Richtung ihres justiziellen Hilfeauftrags hin.

\footnotetext{
S. Kühne $(\bowtie) \cdot$ C. Schlepper

Institut für Soziale Arbeit und Sozialpolitik, Universität Duisburg-Essen,

Universitätsstraße 2, 45141 Essen, Deutschland

E-Mail: sylvia.kuehne@uni-due.de

C. Schlepper

E-Mail: christina.schlepper@uni-due.de
} 


\title{
"It's my duty to write a report"—-Die sanften Kontrolleure" and Their Documents
}

\begin{abstract}
From the perspective of institutional ethnography and drawing upon data material from the replication study "Die sanften Kontrolleure", the article examines how social workers in the field of youth court assistance deploy documents in interaction with their addressees. The results reveal that documents, on the one hand, serve to prevent conflicts and that they assist social workers to communicate their intention to help their addressees. On the other hand, we identified functions which are embedded in strategies of finding the truth. In these cases, documents are primarily used to assist the court. In varying degrees, the file functions as an ally in the interaction and augments the repertoire of social workers for presenting themselves as aid workers-yet not only for their addressees but also for the judicial system. Therefore, the results indicate that, firstly, the inclusion of documents by social workers carries the risk that they are standardizing their action by anticipating a judicial logic of action. Secondly, the diachronic comparison of references on documents in talks between social workers and their addressees points to a shift of the self-positioning of social workers towards an understanding of "judicial aid workers".
\end{abstract}

\section{Einleitung}

In der Tradition der Ethnomethodologie sind Akten bereits früh Gegenstand der Erforschung von Bürokratie (vgl. z.B. Garfinkel 1967, 1974; Cicourel 1968; Smith 1974) und im Hinblick auf ihre Funktion, institutionelle Entscheidungen vorzubereiten und zu legitimieren, untersucht worden. Mit Blick auf ihre Entstehung, Rezeption und Instrumentalisierung gerieten sie insbesondere als Kontrollinstrumente des institutionellen Alltags nicht nur von Medizin und Justiz, sondern auch der Sozialarbeit als Kooperationspartnerin letzterer in den Blick, und schärften diesen für die im Rahmen der dokumentarischen Konstitution von „Fällen“ erfolgenden Kategorisierungsund Etikettierungsprozesse, mithin die „Produktion von Abweichungstatbeständen“ (Wolff 2005, S. 504). Im Kontext einer kritischen Institutionenforschung wurden Akten von Sozialarbeiter_innen daher früh als ,Dokumente der Interventionstätigkeit“" (Cremer-Schäfer 2012, S. 140) wahr- und zum Ausgangspunkt genommen, die „Erzeugungsregeln“ (ebd., S. 141) zu untersuchen, die der Herstellung der dokumentierten „Wirklichkeit“" zugrunde liegen:

Akten dokumentieren die Entscheidungen der „Verwalter“ und stellen sie (retrospektiv) als „,rechtmäßige“ und ,richtige“ Reaktion auf die Natur des Eingriffstatbestandes dar. Aktenanalysen (von Jugendamtsakten, Akten der Familienfürsorge, der Jugendgerichtshilfe, der Obdachlosenhilfe) zielten darauf, Entscheidungsprozesse und -praktiken zu rekonstruieren sowie anhand der Kategorisierungen (Diagnosen und Etiketten) die ,pragmatischen Theorien“ (das ,,implizite Wissen“") der Akteure Sozialer Arbeit herauszuarbeiten. (ebd., S. 140) 
Vor diesem Hintergrund gaben in den 1970er Jahren Auswertungen von Akten von Jugendämtern (vgl. Peters und Cremer-Schäfer 1975, S. 2 mit Bezug auf Haferkamp und Meier 1972) Anlass zu der Vermutung, dass Sozialarbeiter_innen nicht vor dem Hintergrund eines professionellen Selbstverständnisses agieren, in dessen Mittelpunkt ein Hilfeverständnis im originären Interesse ihrer Adressat_innen stehe. Waren die Akten danach ,,voll von Zuschreibungen personalisierender und kriminalisierender Abweichungsetiketten“ (Cremer-Schäfer 2018, S. 23), betonten Peters und Cremer-Schäfer (1975) gleichwohl, dass sich Akten ohne Kenntnis ihrer Entstehungssituation nicht hinreichend erschließen und insofern entlang von Aktendokumentationen kaum Aussagen über das professionelle Selbstverständnis von Fachkräften der Sozialen Arbeit zu treffen seien. Demgegenüber beriefen sie sich auf die interaktionistische Prämisse, dass Verhalten durch die Interpretationen der Handelnden gesteuert und somit auch das Selbstverständnis von Sozialarbeiter_innen als Helfer_innen auf diese Weise determiniert werde. In ihrer Untersuchung „Die sanften Kontrolleure“ (1975), die bis heute als zentrale Referenzstudie gilt, wenn es um Fragen des Umgangs von Fachkräften Sozialer Arbeit mit Devianten und der Ausübung sozialer Kontrolle, aber auch nach ihrem professionellen Selbstverständnis geht, stellten sie den in den Akten formalisierten Kooperationsbeziehungen mit anderen Instanzen sozialer Kontrolle das ,situierte Handeln“ von Sozialarbeiter_innen gegenüber (Cremer-Schäfer 2012, S. 145 ff.). Die Ergebnisse ihrer Studie zu den Interaktionsstrategien von Fachkräften der Sozialen Arbeit untermauerten ihre Annahme, dass sich Dokumente wie Akten nicht nur nicht als objektive Repräsentationen eines Falles, sondern auch nicht von professionellen Selbstverständnissen lesen lassen. Sie zeigten, dass die Aufrechterhaltung der Helfer_innenrolle nicht unwesentlich von den Reaktionen der Adressat_innen auf die Handlungen und die sprachlichen Äußerungen der Fachkräfte abhängig ist, wodurch wiederum das sozialarbeiterische Handlungsrepertoire eingeschränkt wird, da zu diesem Zweck Konflikte vermieden werden und die Hilfeabsicht die Interaktion bestimmt (Peters und Cremer-Schäfer 1975, S. 23). Insofern üben Sozialarbeiter_innen zwar soziale Kontrolle aus. Zum Zweck der Aufrechterhaltung ihres Helferselbstverständnisses tun sie dies jedoch überwiegend in ,sanfter Weise“, d.h. unter Rückgriff auf Interaktionsstrategien, die ihre Adressat_innen ,nicht als deviant etikettieren“ (ebd., S. 24).

Die seit Mai 2016 von der Deutschen Forschungsgemeinschaft geförderte „Vergleichende Replikationsstudie: Die sanften Kontrolleure“" zielt darauf ab, zu prüfen, ob die theoretischen Annahmen und empirischen Befunde mehr als vierzig Jahre später noch Bestand haben. Wenngleich die Studie von Peters und Cremer-Schäfer insbesondere die Diskrepanz zwischen dokumentarischer Wirklichkeit und situiertem Handeln - den Interaktionsstrategien - von Fachkräften Sozialer Arbeit mit ihren Adressat_innen fokussierte, nehmen wir, basierend auf unseren Beobachtungen, in unserer Analyse für diesen Beitrag ergänzend den Einfluss des Prinzips der Schrift-

\footnotetext{
1 An dem Projekt (gefördert durch die Deutsche Forschungsgemeinschaft (DFG) - Projektnummer 289782537; Laufzeit: 34 Monate) sind - neben den beiden Autorinnen - Jan Wehrheim als Projektleiter sowie Margarete Killian und Lisa Paffrath als studentische bzw. wissenschaftliche Hilfskraft beteiligt.
} 
lichkeit auf Gespräche zwischen Sozialarbeiter_innen und ihren Adressat_innen in den Blick.

In Anlehnung an die Systematik, die Lindsay Prior (2008a, 2008b) zu den Potenzialen der Dokumentenanalyse vorgelegt hat, sind wir weniger an den Dokumenten in ihrer Funktion als Informationsquellen interessiert und auch nicht an der Frage, wie z. B. Akten durch unterschiedliche Akteure hervorgebracht werden. Wir betrachten sie demgegenüber in ihrer Beziehung zu den Teilnehmer_innen im Gespräch und folgen der Frage, welche Funktionen ihr Vorhandensein resp. ihre Thematisierung in Interaktionen von Fachkräften Sozialer Arbeit mit ihren Adressat_innen annehmen kann.

Nach einer kurzen Beschreibung unseres methodischen Vorgehens sowie der von uns in die Analyse einbezogenen Gesprächstypen und Dokumente zeigen wir, wie Sozialarbeiter_innen Dokumente in der Interaktion mit ihren Adressat_innen zum Einsatz bringen. Im Anschluss betrachten wir unsere Ergebnisse im Spiegel der theoretischen Annahmen und Befunde der Untersuchung von Peters und CremerSchäfer, um Aussagen darüber ableiten zu können, auf welche Kontinuitäten und Diskontinuitäten im Spannungsfeld von Interaktionsstrategien und Helferselbstverständnis die von uns identifizierten Funktionen von Dokumenten hindeuten.

\section{Die empirische Analyse}

\subsection{Methodisches Vorgehen}

Das methodische Vorgehen unserer Replikationsstudie orientiert sich zwar größtmöglich an der Vorgehensweise von Peters und Cremer-Schäfer, d.h. auch wir begleiteten während unserer mehrwöchigen Feldaufenthalte Sozialarbeiter_innen bei ihren Adressat_innenkontakten in den Bereichen der Erziehungshilfe von Jugendämtern und privaten Trägern ${ }^{2}$ im Rahmen von Gesprächsterminen oder Sprechstunden, Außenterminen sowie Hausbesuchen, und protokollierten ihre Gespräche. Anders als Peters und Cremer-Schäfer und in Orientierung an den Prämissen der institutionellen Ethnographie (Smith 2005) führten wir jedoch auch Interviews mit einigen der beobachteten Sozialarbeiter_innen, um neben den Interaktionspraktiken Einblick in ihre Ansichten über ihr Handeln sowie ihr professionelles Selbstbild zu gewinnen. Darüber hinaus erhoben wir Dokumente, die die Arbeit in den Einrichtungen prägen und die wir dort vorfanden: sowohl Akten insgesamt als auch speziell Anklageschriften, Polizeiberichte, Berichte der Jugendgerichtshilfe (JGH-Berichte), Trägerberichte, Fragebögen, Protokolle und Risikoeinschätzungsbögen. Diese Do-

\footnotetext{
${ }^{2}$ Die verstärkte Heterogenität der Felder und Institutionen Sozialer Arbeit verlangte eine Ausweitung der Untersuchung. Anders als Peters und Cremer-Schäfer (1975), die Jugendämter und private Fürsorgeeinrichtungen in zwei Städten, die entlang der Dimensionen konfessionelle Mehrheit und politisches Wahlverhalten divergierten, miteinander verglichen, ist unsere Auswahl vor allem an institutionellen Merkmalen der Einrichtungen orientiert, die sich vor dem Hintergrund vielfältiger Transformationen im postwohlfahrtsstaatlichen Kontext (Kessl und Otto 2009; Kessl 2013) beobachten lassen und Anlass zu der Vermutung geben, dass damit auch die Interaktionspraktiken von Fachkräften Sozialer Arbeit variieren.
} 
kumente bilden mit Blick auf ihre Verwendung in den Gesprächen den Gegenstand unserer Analyse für diesen Beitrag.

Dokumente bzw. Dokumentationsordnungen (Akten), d.h. „schriftliche Texte, die als Aufzeichnung oder Beleg für einen Vorgang oder Sachverhalt dienen“ (Wolff 2005, S. 502), lassen sich als ,institutionalisierte Spuren“ (ebd., S. 503) lesen. Im Rahmen der Dokumentenanalyse ermöglichen sie Zugang zu den sich in ihnen abbildenden oder diese beeinflussenden (Prozessen der) Fall-, d.h. Wirklichkeitskonstruktionen. Vor diesem Hintergrund bildet unseren Ausgangspunkt, dass ebenso wie die Untersuchung der „dokumentarischen Wirklichkeit“ (Wolff 2005, S. 502) Aufschluss über die soziale Organisation ihrer Herstellung geben kann, Dokumente in gleichem Maße ein ,interaktives, personenbezogenes (soziales) Verhältnis“ (Muckel 1997, S. 11 mit Bezug auf Akten, Herv. weggelassen) zu ihren Produzenten und Objekten konstituieren: Gerade weil sie ein allgegenwärtiger Bestandteil der Praxis Sozialer Arbeit sind, entlang unterschiedlicher Schnittstellen resp. Situationen entstehen und sich verändern, besitzen sie für Fachkräfte der Sozialen Arbeit sowohl im Kontakt mit ihren Adressat_innen als auch im Austausch mit Organisationen oder Behörden Relevanz. Dokumente dienen im Hilfeprozess z. B. der Planung und Steuerung von Hilfen oder auch der Strukturierung und Bewertung von Hilfeverläufen und -ergebnissen (vgl. Merchel und Tenhaken 2015, S. $171 \mathrm{f}$.). Ihre Funktionen reichen von der Erinnerung innerhalb zunehmend komplexerer Arbeitswelten bis hin zur Legitimation und Evaluation (vgl. Ley und Seelmeyer 2014, S. 51; Ley 2010; Taylor und White 2000, S. 141 ff.). In dieser Perspektive werden Dokumente als Arbeitsgegenstand von Fachkräften Sozialer Arbeit in Augenschein genommen und im Hinblick auf ihre Funktionen, etwa als Lese- und Handlungsanleitung sowie als Instrumente der internen und externen Handlungskoordination, betrachtet (Nadai 2015, S. 253). Dabei geht es, in anderen Worten, um die Bedeutung, die textförmigen Dokumenten im Hilfeprozess zukommt. Diese Frage verfolgen wir in diesem Beitrag auch. Statt jedoch das Hauptaugenmerk darauf zu legen, wie in Dokumenten vorgenommene institutionelle Setzungen das Handeln der Fachkräfte strukturieren (vgl. Nadai 2012), greifen wir die in dieser Forschungsrichtung bislang eher randständige Frage nach dem Einfluss von schriftlicher Dokumentation auf das interaktive Handeln zwischen Sozialarbeiter_innen und Adressat_innen auf. Dabei fokussieren wir, dem Anliegen unserer Replikationsstudie folgend, vor allem die von den Fachkräften zur Anwendung gebrachten Interaktionspraktiken im Zusammenhang mit ihrem Potential, Devianz zu ,produzieren“. ${ }^{3}$ Steht insofern die Relevanz der Dokumente im Hinblick auf ihre symbolisch-kommunikative als auch

\footnotetext{
3 Zwar existieren neuere Untersuchungen, die den „Akteurs“-Beitrag von Dokumentationen im Kontext sozialarbeiterischen Handelns analysieren. Diese nehmen jedoch im Zusammenhang mit der Mediatisierung (in) der Sozialen Arbeit vor allem das zunehmend medial vermittelte Arbeiten in den Blick, d.h. den Einfluss der Dokumentation durch IT-gestützte Anwendungen (z. B. Kutscher et al. 2015) und tragen damit der Entwicklung Rechnung, dass Fachsoftware zu einem Instrument der Falldokumentation und Hilfeplanung in zahlreichen Handlungsfeldern der Sozialen Arbeit geworden ist (vgl. Ley und Seelmeyer 2014). Dabei konzentriert sich die empirische Forschung vor allem auf das Handlungsfeld der Arbeitsverwaltung. Im Mittelpunkt steht die Frage, wie am PC zu erstellende Dokumente, z. B. die Eingliederungsvereinbarung (Böhringer 2015), in das Gespräch integriert werden. Fokussiert wird insofern der Einzug der Technik in die Praxis Sozialer Arbeit, die in Form der Medien Eingang in den sozialarbeitswissenschaftlichen Diskurs gefunden hat.
} 
praktisch-materielle Wirkmacht (vgl. Braun-Thürmann 2006) in den alltäglichen Interaktionen von Fachkräften Sozialer Arbeit im Mittelpunkt, korrespondiert dies mit dem Kernanliegen der Workplace Studies, die sich dafür interessieren, ,wie [diese] Instrumente und Technologien in sozialen Handlungen und Interaktionen verwendet werden." (Knoblauch und Heath 1999, S. 168, Herv. hinzugefügt) Hierfür bedienen wir uns der Perspektive der institutionellen Ethnographie (Smith 2005), wonach Dokumentationen als Mittel sozialarbeiterischer Interventionstätigkeit auch dahingehend betrachtet werden können, dass sie, eingebettet in komplexe Interaktionskontexte - etwa zwischen Fachkräften und Adressat_innen -, auch auf Kommunikation zurückwirken und bezüglich ihres Einflusses auf das Prinzip der Mündlichkeit selbst untersucht werden können.

In der Analyse für diesen Beitrag beschränken wir uns, erstens, auf die insgesamt 74 Gespräche, ${ }^{4}$ die wir in zwei Einrichtungen der Jugendgerichtshilfe (JGH) während zwei Feldaufenthalten von jeweils fünf Wochen beobachtet haben ${ }^{5}$ sowie auf die von Peters und Cremer-Schäfer protokollierten Gespräche, in denen die von ihnen beobachteten Fachkräfte ihren Adressat_innen in der Rolle als Jugendgerichtshelfer_innen gegenüber traten. ${ }^{6}$ Entgegen fortschreitender Tendenzen der Mediatisierung und Digitalisierung in anderen Handlungsfeldern der Sozialen Arbeit (Kutscher et al. 2015; Kutscher 2018) legen unsere Beobachtungen in der Jugendgerichtshilfe nahe, dass die digitale Aktenführung längst nicht flächendeckend die traditionelle Papierakte oder traditionelle Dokumentationen abgelöst hat. In den beobachteten Einrichtungen der Jugendgerichtshilfe ist die „Potentialität des Einflusses der IT“ (Ley 2010, S. 221, Herv. weggelassen) insgesamt als vergleichsweise gering auszuweisen. ${ }^{7}$

\footnotetext{
${ }^{4}$ Wie die Autor_innen der Originalstudie haben auch wir uns dafür entschieden, die Gespräche handschriftlich zu protokollieren, da wir vermuten, dass Sozialarbeiter_innen und Adressat_innen unsere Anwesenheit bei Audioaufnahmen häufiger ablehnen würden. Damit geht ein Genauigkeitsverlust einher, der den Verzicht auf konversations- oder sequenzanalytische Auswertungsverfahren notwendig macht.

5 In den Einrichtungen der Jugendgerichtshilfe waren wir in der Regel von 8 oder 9 Uhr morgens bis ca. 17 Uhr vor Ort und beobachteten Gespräche, die in den Büros der Sozialarbeiter_innen stattfanden. Unser Ziel war, diese Gespräche möglichst wörtlich zu protokollieren. Hierzu saßen wir fast immer mit am Besprechungstisch, nur bei einer Sozialarbeiterin konnten wir an einem Schreibtisch hinter einer großen Grünpflanze sitzen. Da die Fachkräfte sehr unterschiedlich mit unserer Präsenz umgingen - mal wurde den Adressat_innen der Zweck unserer Anwesenheit vor dem Hintergrund der Studie erläutert, mal nur ein Hinweis gegeben, dass es sich um eine Kollegin handle, mal wurde unsere Gegenwart gar nicht thematisiert -, haben wir zwar immer eine teilnehmende, aber variierend eine offen und eine (zumindest gegenüber den Adressat_innen) verdeckt beobachtende Forscherinnenrolle eingenommen. Obwohl die Umgangsweisen mit unserer Anwesenheit und Rolle durch die begleiteten Sozialarbeiter_innen, von denen wir mit sieben gegen Ende unserer Feldphasen Leitfadeninterviews führten, unterschiedlich waren, konnten wir nicht feststellen, dass sich diese auf das Verhalten der Adressat_innen in der Gesprächssituation auswirkten.

${ }^{6}$ An dieser Stelle gilt unser Dank Helga Cremer-Schäfer, die uns die Gesprächsprotokolle der Studie zur Verfügung gestellt hat.

7 Dass die Technik in den von uns beobachteten Einrichtungen im Vergleich zu anderen Handlungsfeldern mit eigenen Software-Anwendungen (ASD, Bewährungshilfe, Arbeitsvermittlung) bislang wenig institutionalisiert ist, zeigt sich auch daran, dass die Fachkräfte zwar über eigene PCs verfügen, davon aber nicht Gebrauch machen müssen, um Berichte zu schreiben, da sie die Möglichkeit haben, sie auf Band zu sprechen und von Schreibkräften tippen zu lassen.
} 
Mit Blick auf die nachfolgend dargestellten Typen von Gesprächen im Feld der JGH ist unser Forschungsgegenstand, zweitens, auf zwei Arten von Dokumenten beschränkt: Jene, die entweder während der Interaktion bereits vorliegen und als „documents in use“ (Prior 2008b, S. 489) daran beteiligt sind oder deren Erstellung die Interaktion dient. ${ }^{8}$ In anderen Worten berücksichtigen wir Akten, die in der Regel Anklageschriften und Polizeiberichte enthalten, und JGH-Berichte.

\subsection{Gesprächstypen und Dokumente im Feld der Jugendgerichtshilfe}

Gespräche zwischen Sozialarbeiter_innen und Adressat_innen im Handlungsfeld Jugendgerichtshilfe sind durch eine spezifische ,interaktive Ordnung institutioneller Aktivitäten“ (Hitzler und Meßmer 2008, S. 246; Herv. i. O.) gekennzeichnet, entlang derer sich zwei typische Formen von Gesprächen ausmachen lassen: In diesem Setting unterscheiden sich die institutionellen Aktivitäten je nachdem, ob es sich um ein Gespräch zur Vorbereitung einer Gerichtsverhandlung handelt oder um ein Gespräch, das im Kontext der Diversion stattfindet, da ihnen teilweise verschiedene Handlungsaufträge zugrunde liegen. ${ }^{9}$ Diese institutionellen Gesprächstypen, wie sie sich im gesprächsanalytischen Sinne bezeichnen lassen (Stukenbrock 2013, S. 223), sind in den von uns untersuchten Einrichtungen in Prozessschritte resp. Ziele untergliedert, welche in sog. Schlüsselprozessen festgeschrieben sind und das Handeln der Sozialarbeiter_innen anleiten (sollen). So besteht die Aufgabe der Fachkraft im ersten Gesprächstyp darin, die Adressat_innen umfassend über den Ablauf der Hauptverhandlung sowie ihre Rechte und Pflichten zu informieren und die für den JGH-Bericht erforderlichen Daten zu erfragen. Hierfür benötigen sie Auskünfte über die Lebens- und Wohnsituation, die schulische und ggf. berufliche Laufbahn, die Familienverhältnisse, die finanzielle Situation und das Freizeitverhalten der Adressat_innen. Darüber hinaus enthält der Bericht eine Stellungnahme zur Persönlichkeitsentwicklung und strafrechtlichen Verantwortungsreife sowie ggf. auch einen Vorschlag zu Reaktionsmöglichkeiten des Gerichts („Maßnahmenvorschlag“). Dies muss zwar nicht zwingend mit den Adressat_innen erörtert werden, das Gespräch dient der Fachkraft jedoch als Grundlage, sich darüber eine Meinung zu bilden.

Der zweite Gesprächstyp enthält die Prozessschritte resp. Ziele, das den Adressat_innen vorgeworfene strafbare Handeln zu erörtern sowie die Einsicht in das Fehlverhalten zu fördern und ggf. eine Diversionsmaßnahme aufzuerlegen. Zwar obliegt Sozialarbeiter_innen auch in Diversionsverfahren eine Berichtspflicht, denn sie müssen einen Diversionsbericht für die Staatsanwaltschaft erstellen. Dieser erfordert jedoch keine detaillierte Wiedergabe der Lebensläufe von Adressat_innen und das

\footnotetext{
${ }^{8}$ Insofern beziehen wir uns nicht nur auf Dokumente, die in den Gesprächen physisch vorliegen, sondern auch auf solche, die in Anlehnung an techniksoziologische Begrifflichkeiten als „Quasi-Objekte“ (Serres 1987; Latour 1995) vorhanden sind. Wie im Fall des Jugendgerichtshilfeberichtes konstituieren sie den Anlass des Gesprächs, d.h. sie sind, ohne bereits vorzuliegen, Gegenstand der Auseinandersetzung zwischen Sozialarbeiter_innen und Adressat_innen.

9 Da wir Gespräche aus der Analyse ausgeklammert haben, die der Durchführung einer Diversionsmaßnahme dienen (z. B. Buchprojekt, Täter-Opfer-Ausgleich), wird dieser Gesprächstyp nicht näher beschrieben.
} 
Gespräch fungiert in den Schlüsselprozessen - anders als beim ersten Gesprächstyp - nicht als Berichtsgrundlage. Insofern sind den Sozialarbeiter_innen unterschiedliche Handlungsrahmen vorgegeben, die mit jeweils typischen Gesprächsverläufen einhergehen. Empirisch lassen sich jedoch durchaus auch Mischformen feststellen.

In beiden Gesprächstypen stellt die Ermittlungsakte, ${ }^{10}$ die die Sozialarbeiter_innen von der Staatsanwaltschaft zugeschickt bekommen, ein Objekt dar, aus dem sich nicht nur das Vorwissen der Fachkraft über „den Fall“" speist, sondern das auch strukturierend auf das Gespräch einwirken kann. ${ }^{11}$ Die Akte enthält in der Regel die Anklageschrift und Polizeibericht(e), also Dokumente, die von den Strafverfolgungsbehörden angefertigt wurden, ihren institutionellen Logiken dienen und entsprechend gefärbte „Fiktionen“ (Lau und Wolff 1981, S. 208) des Tatgeschehens ihrer Verfasser_innen wiedergeben. Insofern kann diese spezielle Zurichtung des Falls in der Ermittlungsakte die Wahrnehmung der Sozialarbeiter_innen prägen. Dies legt die Annahme nahe, dass je mehr sich die Sozialarbeiter_innen im Gespräch an der Akte und dem in ihr festgehaltenen verdinglichten Etikett orientieren, sie die institutionelle Logik der Strafverfolgungsbehörden antizipieren, und sich dies in ihrer Handlungspraxis niederschlägt. Vor dem Hintergrund dieser These analysierten wir in den von uns ausgewerteten Gesprächsprotokollen jene Passagen, in denen verschiedene Dokumentenarten thematisch wurden. Es geht uns in unserer Untersuchung nicht darum herauszufinden, ob ein bestimmtes Gespräch gut oder schlecht verlief oder ob sich die einzelnen Fachkräfte in einer Situation angemessen oder unangemessenen verhielten, sondern ,,[b]ei der Gesprächsanalyse interessiert, wie Gesprächsteilnehmer interaktiv relevante Realität konstituieren" (Deppermann 2008, S. 79, Herv. i.O.). ${ }^{12}$ Vor diesem Hintergrund waren die Suchbewegungen unserer Datenanalyse unter anderem von folgenden Fragen geleitet: Wie wird die Interaktionsaufgabe von den Beteiligten bewältigt? Welche Teilaktivitäten gibt es und in welchem Verhältnis stehen sie zur Aufgabe? Die typischen Muster, die sich im Hinblick auf die spezifischen Funktionen der (Thematisierung von) Dokumenten in der Interaktion identifizieren ließen, stellen wir im Folgenden vor.

\subsection{Dokumente in Interaktion}

Die Abgrenzung einer Analyse von „Documents-in-Interaction“ von ihrer Analyse als „Behälter von Inhalten“ bzw. ,,in Aktion“ ist, wie es Prior (2008a, S. 480) formuliert hat, weniger eine empirische als vielmehr eine konzeptionelle. Mitunter kann die Frage danach, was Dokumente tun, auch mit der Frage zusammenhängen, was diese „sagen“, d.h. was ihr Inhalt ist - ein Zusammenhang, der sich bereits im Hin-

\footnotetext{
10 Die Akten selbst waren nicht Gegenstand unserer Untersuchung. Wir konnten diese lediglich sporadisch einsehen.

11 Wie Merchel (2004, S. 23) anmerkt, obliegt es der jeweiligen Fachkraft, wie sie die Akte im Kontext der Sinnbezüge ihrer Organisation nutzt.

12 Die Anlehnung unserer Auswertungsmethodik an die von Deppermann (2008) vorgelegte Methode der Gesprächsanalyse erlaubt es, in den Protokollen dominante Gesprächsmuster, Konversationspraktiken und Gesprächskonflikte zu identifizieren sowie Strukturen thematischer Abläufe nachzuvollziehen.
} 
blick auf die mutmaßlich augenscheinlichste Funktion von Dokumenten andeutet: die Gesprächsstrukturierung.

\subsubsection{Gespräche strukturieren}

Unseren Beobachtungen zufolge dient insbesondere die Thematisierung des JGHBerichts einerseits dazu, die interaktive Ordnung des Gesprächs herzustellen. Dies geschieht in der Regel bereits bei der Gesprächseröffnung, wie dieser Auszug aus einem Gesprächsprotokoll (Prot. 70, 8-10) ) $^{13}$ verdeutlicht. Vermittelt über seine Berichtspflicht kündigt der Sozialarbeiter dem Adressaten in diesem Beispiel ohne Umschweife das von ihm erwartete Verlaufsmuster des Gesprächs an:

Herr Arslan: ${ }^{14}$ Meine Aufgabe ist, für die Staatsanwaltschaft und den Richter einen Bericht zu schreiben. Das, was du mir erzählst, schreibe ich in den Bericht. Bei Fragen können wir uns später darüber unterhalten. Haben Sie oder hast du auch diese Gerichtsmitteilung bekommen?

Adressat: Ja.

Herr Arslan: Bis dahin sollte oder muss mein Bericht vorliegen. Bevor wir darüber sprechen, brauche ich einige Informationen über [nennt einige Gliederungspunkte des Berichts]. Ich frage, Sie oder du antwortest.

Andererseits kann der JGH-Bericht nicht nur in struktureller, sondern auch in inhaltlicher Hinsicht in dem Maße zur zentralen Richtstruktur der Gesprächsorganisation werden, wie seine Gliederungspunkte in der darauf folgenden Gesprächsphase als Stichwortgeber in der Interaktion fungieren. Entlang der im vorigem Abschnitt erwähnten typischen Inhalte des Berichtes werden in den Gesprächen dann etwa Fragen zu den familiären Verhältnissen der Adressat_innen gestellt, Informationen zu Kindergarten- und Schulbesuchen erhoben, das Freizeitverhalten oder auch die Einstellung zur Tat erfragt. Daran können sich Erörterungen anschließen, die schlussendlich die sozialarbeiterische Stellungnahme zur strafrechtlichen Verantwortungsreife und Sozialprognose sowie Vorschläge zur aufzuerlegenden Maßnahme zum Inhalt haben. Exemplarisch hierfür steht ein Gespräch zwischen einer Sozialarbeiterin und einem Jugendlichen (Prot. 65, 4-102), der wegen Fahrerflucht angeklagt ist, in welchem erstere sukzessive die Berichtsdaten erfragt:

Frau Dreher: Es geht um die Sache mit der Unfallflucht am [Datum]. Da Sie noch keine 21 sind, sondern 19 waren, kommen Sie nochmal vor's Jugendgericht. Meine Aufgabe ist, einen Bericht zu schreiben. Gegebenenfalls können Sie was zur Sache sagen, ich sage etwas dazu, ob Jugend- oder Erwachsenenstrafrecht angewendet werden soll und mache einen Vorschlag zur Maßnahme. Ich muss etwas über Ihren Lebenslauf schreiben. Wie heißt der Vater?

\footnotetext{
13 Das dargestellte Kürzel kennzeichnet die im Verlauf der Feldphasen chronologisch vergebene Protokollnummer sowie den jeweils zitierten Protokollauszug entsprechend der fortlaufenden Zeilennummerierung des Protokolls.

14 Um Rückschlüsse sowohl auf die untersuchten Einrichtungen als auch die in ihnen tätigen Fachkräfte Sozialer Arbeit auszuschließen, wurden sämtliche Namen der Jugendgerichtshelfer_innen in diesem Beitrag zu Darstellungszwecken pseudonymisiert.
} 
Adressat: [Vorname Nachname]

Frau Dreher: Wann ist er geboren?

Adressat: [Datum]

Frau Dreher: Was macht er beruflich?

Adressat: Gerüstbauer. (4-9)

[Es folgen Fragen der Sozialarbeiterin zu Geschwistern, der Migrationsgeschichte, Kindergarten- und Schulbesuchen sowie der beruflichen Ausbildung] Frau Dreher: Haben Sie direkt nach dem [Berufs- und weiterführende Schule] nach einer Ausbildung geguckt?

Adressat: Ja, da haben wir viele Praktika gemacht, zwei im Jahr, Handwerk und Klimatechnik hat mir nicht so gefallen.

Frau Dreher: Was machen Sie in Ihrer Freizeit?

Adressat: Viel Sport, schwimmen.

Frau Dreher: Im Verein?

Adressat: Ja, Schwimmverein. (54-59)

[Es folgen Fragen der Sozialarbeiterin zum Tathergang]

Frau Dreher: Keine Schulden. Sollte das Gericht zu der Auffassung kommen, dass Sie das [den Unfall] bemerkt haben hätten müssen, würde ich Jugendstrafrecht vorschlagen. Sie haben ja noch keine Ausbildung. Maßnahme könnte Geldbuße oder Arbeitsstunden sein. (102)

In den durch diesen expliziten Bezug auf den JGH-Bericht charakterisierten Fällen zeigt sich eine starke Institutionalisierung der Interaktion (die, wie später zu zeigen sein wird, auch in Bezug auf Inhalte der Akte auszumachen ist), welche mitunter am Gesprächsende ihre finale Rahmung erhält, wenn, wie in nachfolgendem Beispiel (Prot. 54, 126-128), Sozialarbeiter_innen das Gespräch mit dem Hinweis darauf, nun den Bericht fertigzumachen, abschließen:

Frau Dreher: Haben Sie noch Fragen?

Adressat: Nein.

Frau Dreher: Gut, dann werd' ich den Bericht fertigmachen.

Im Hinblick auf diese Funktion, das Gespräch zu strukturieren, lassen sich in unserem Material Unterschiede identifizieren - sowohl im Vergleich zwischen den untersuchten Einrichtungen der Jugendgerichtshilfe als auch zwischen beobachteten Sozialarbeiter_innen derselben Einrichtung: Während etwa in der Einrichtung des freien Trägers alle Fachkräfte ihre Gespräche entlang des Berichtes strukturieren, weichen in der von uns untersuchten städtischen Jugendgerichtshilfe Sozialarbeiter_innen, vor allem bei Diversionsgesprächen, davon ab. Deutet dies auf unterschiedliche Einrichtungskulturen hin, variiert auch der Einfluss, den die Thematisierung des Berichts und mithin der Berichtspflicht auf die Standardisierung des Gesprächs auf der individuellen Ebene hat: Während manche Fachkräfte einen stark standardisierten Gesprächsstil zeigen, in dem die Abfolge der im Bericht zu erfassenden Informationen in hohem Maße das Gesprächsverhalten steuert, passen andere Sozialarbeiter_innen diesen individuell der Situation an - mit der Folge, dass die Orientierung am Bericht partiell verdeckt oder implizit bleibt. 
Unabhängig vom Ausmaß dieser Standardisierung birgt jedoch auch die implizite Orientierung an den Berichtsinhalten das Risiko in sich, allgemeine Höflichkeitsregeln $\mathrm{zu}$ brechen, weil zuweilen gesichtsbedrohende Themen elaboriert werden müssen. So besteht die Notwendigkeit, Sachverhalte zu eruieren, die es, in der Terminologie von Erving Goffman (1974, S. 68 ff.) erforderlich machen, die „Informations“- und „Gesprächsreservate“ der Adressat_innen in einer der Situation eigentlich unangemessenen Weise zu betreten. Fragen der Fachkräfte, etwa nach partnerschaftlichen Verhältnissen im Rahmen der Erhebung des Freizeitverhaltens oder nach dem Tatgeschehen, können Beschämungen der Adressat_innen nach sich ziehen. Damit entsteht nach Garfinkel (1956) ein gewisses Degradationsrisiko, welches, folgt man den Befunden der Studie von Peters und Cremer-Schäfer (1975), ein Risiko für die Aufrechterhaltung der Helfer_innenrolle darstellt und insofern den Zielen sozialarbeiterischen Handelns zuwiderlaufen könnte.

\subsubsection{Gesichtsbedrohende Themen neutralisieren}

Vor diesem Hintergrund deuten unsere Befunde darauf hin, dass der Verweis auf Informations- oder Dokumentationspflichten ebenso wie auf vorliegende Dokumentationen auch dazu dienen kann, dieses Risiko zu entschärfen und insofern das daraus resultierende „Dilemma auf der Interaktionsebene“ (Hitzler und Meßmer 2008, S. 249) zu vermeiden. In unserem Datenmaterial finden sich Gespräche, in denen sich eine weitere Funktion der Thematisierung von Dokumenten darin zeigt, dass Sozialarbeiter_innen potenziell gesichtsbedrohende Gesprächsinhalte neutralisieren, indem sie diese auf eine Weise einleiten, die den Fortgang des Gesprächs nicht gefährdet - etwa dadurch, dass Gesprächsanlässe und Vorgänge lediglich auf der behördlichen Ebene thematisiert werden. Dies kann, wie im folgenden Beispiel eines Gesprächsbeginns, in der Form geschehen, dass die Akte als Symbol für die Sache fungiert, d.h. Fachkräfte den Anlass des Gesprächs einführen, ohne gleichsam das strafrechtliche Label aus der Akte zu übernehmen. In diesem Beispiel beginnt der Sozialarbeiter das Gespräch mit den Worten (Prot. 06, 5):

Herr Matthäus: Die Staatsanwaltschaft hat mir die Akte geschickt und mich gebeten zu prüfen, ob man die Sache einstellen kann.

Ähnlich wird auch der „Papierweg“, den eine mögliche Straftat nach sich zieht, statt die strafbare Handlung selbst angesprochen (Prot. 26, 6):

Herr Matthäus: Ich hab' leider wieder 'ne Anklageschrift bekommen.

Das mit der Thematisierung des Tatvorwurfs und der Abfrage der Berichtsdaten einhergehende Risiko der Herabsetzung, das den Ablauf der Interaktion beeinflussen könnte, verweist auch auf ein grundlegendes potenzielles moralisches Dilemma, das sich im Rollenkonflikt der Jugendgerichtshelfer_innen - wie ihn auch die Originalstudie zugrunde legte (Peters und Cremer-Schäfer 1975, S. 21) - manifestiert: das Doppelmandat. Danach sind Sozialarbeiter_innen, einerseits, zwar zur Kooperation mit anderen Instanzen gezwungen und ihnen ist damit auch ein spezifisches Handlungsmuster auferlegt. Vor dem Hintergrund der Annahme eines Helferselbstverständnisses, das auf Hilfe im Sinne der Adressat_innen zielt, müssen sie ihr 
Handeln, andererseits, aber so regulieren, dass ihre Adressat_innen in der Lage sind, die Hilfeabsicht als solche interaktiv anzuerkennen.

\subsubsection{Rollenkonflikte vermeiden}

Interaktionsstrategien, die darauf zielen, auch in dieser Hinsicht Konflikte zu vermeiden, sind unseren Beobachtungen nach ebenfalls mit dem Einsatz von Dokumenten verwoben. So kann der Bezug der Fachkräfte auf Dokumente und mithin die Dokumentationspflicht auch dazu dienen, damit indirekt auf institutionelle Regeln zu verweisen, die das eigene Handeln rechtfertigen und insofern dazu, den Rollenkonflikt zu vermeiden, etwa wenn es darum geht, eine aufzuerlegende Maßnahme in das Gespräch einzubringen. Eine Möglichkeit, eine Diversionsmaßnahme in das Gespräch einzuführen und sich zugleich von dieser zu distanzieren, besteht, wie im folgenden Beispiel, darin, dies unter Verweis auf die Akte zu tun (Prot. 32, 20):

Herr Brüdigam [hat die Akte in der Hand und liest vor]: Das hat der Staatsanwalt geschrieben: Gesprächskurs zum Thema Ladendiebstahl und eine Auflage von 30 Stunden nach Weisung durch das Jugendamt.

Vergleichbares zeigt sich auch, wenn Sozialarbeiter_innen der Berichtpflicht durch das Einholen des Schuldeingeständnisses Genüge tun: Dabei entfaltet der ausdrückliche Bezug auf Informationen aus der Akte einen entlastenden Effekt, der sich darin offenbart, dass er die Sozialarbeiter_innen vom Einsatz von Interaktionspraktiken befreit, die auf die Eruierung von Schuld zielen. Beispielhaft zeigt sich dies in den folgenden Passagen (Prot. 14, 10-12):

Herr Matthäus: Ich hab' die Akte von der Staatsanwaltschaft hier. Da steht in der Anklage drin, dass du bei [großer Supermarkt] ein paar Kopfhörer geklaut hast.

Adressat: Ja.

Herr Matthäus: Du musst zum Tatvorwurf nichts sagen, ich bin Sozialpädagoge und nicht die Polizei. Es gibt die Möglichkeit ein Diversionsverfahren durchzuführen. Das geht bei solchen Bagatelldelikten. Bei schwerwiegenderen Taten gibt es das nicht. Die Voraussetzung dafür ist, dass du geständig bist und einsichtig und dass das 'ne einmalige Tat war. Naja, manchmal sitzen hier auch welche, die das Ganze schon mal gemacht haben. Bei der Polizei hast du ja zugegeben, dass du die Kopfhörer geklaut hast.

Indem sich Herr Matthäus auf die Informationen bezieht, die er der Akte entnehmen kann, konstatiert er das Schuldeingeständnis des Adressaten als bereits etabliertes Faktum, das nicht mehr ausgehandelt werden muss. Auch im folgenden Beispiel verweist der Sozialarbeiter auf das in der Akte dokumentierte bei der Polizei erfolgte Schuldeingeständnis - mit der Folge, dass er dieses von der Adressatin nicht erfragen muss (Prot. 23, 14):

Herr Matthäus: Wie gesagt, du hast das Recht, nichts zu sagen, dann schicke ich die Akte an die Staatsanwaltschaft zurück. Aber wie ich in der Akte sehe, du hast bei der Polizei gesagt, dass es so gewesen ist. 
Die Thematisierung der Dokumente dient in den angeführten Beispielen auch dazu, zu den Kooperationspartner_innen, also in diesem Fall der Justiz, auf Distanz zu gehen - entweder, weil kommuniziert wird, dass den Fachkräften die Berichtspflicht auferlegt ist, oder bestimmte Themen interaktiv erst gar nicht ausgehandelt werden müssen. Gleichwohl zeigt sich in unserem Datenmaterial ebenfalls, dass über die Thematisierung der Akte auch eine Instrumentalisierung dieser Kooperation stattfindet, die wir vor allem in Gesprächen zur Diversion beobachten konnten.

\subsubsection{Kooperationsbereitschaft erwirken}

So besteht eine Taktik, sich die Kooperations- und Auskunftsbereitschaft der Adressat_innen zu sichern, darin, den Eindruck zu erwecken, die Fachkraft erspare dem bzw. der Jugendlichen die Gerichtsverhandlung durch das Anfertigen des JGH-Berichts (und nicht durch das Auferlegen oder Absehen von einer Diversionsmaßnahme). Die folgende Gesprächssequenz, in der der Adressat wegen Marihuanabesitz angezeigt wurde (Prot. 61, 4-7), illustriert diese Funktion der Thematisierung des JGH-Berichts:

Frau Dreher: Sie waren beim Fußballspiel.

Adressat: Mhm.

Frau Dreher: Da hat man bei Ihnen einen Joint festgestellt. Die Polizei hat das an die Staatsanwaltschaft weitergeben und die hat die Akte hierher geschickt mit der Bitte um Diversion, also keine Gerichtsverhandlung, um das hier aus der Welt zu schaffen. Dafür muss ich etwas über Ihren Lebenslauf schreiben.

Adressat: Ok.

Zunächst rekonstruiert Frau Dreher anhand der Aktenlage den dokumentierten Tathergang und zeichnet den bisherigen Verfahrensverlauf durch die involvierten Institutionen sozialer Kontrolle nach, um das Verfassen des Berichts als Bedingung, um „das hier aus der Welt zu schaffen“, zu formulieren, womit sie die Räume der Jugendgerichtshilfe anstelle des Gerichtssaals meint. Folgt man der Originalstudie, dann gefährden Kooperationen von Sozialarbeiter_innen mit der Polizei oder den Justizbehörden das Vertrauensverhältnis zu den Jugendlichen. Demgegenüber zeigt dieses Beispiel, dass das Reglement zur Anfertigung des JGH-Berichts, das der justizielle Handlungsauftrag mit sich bringt, vielmehr auch zum „Instrumentalisieren des moralischen Dilemmas“ (Hitzler und Meßmer 2008, S. 249 mit Bezug auf Hydén 1994) genutzt werden kann.

Äußerungen in Bezug auf vorliegende oder zu erstellende Dokumente erweisen sich vor diesem Hintergrund als multifunktional und die angeführten Funktionen verdeutlichen, dass sie sowohl in struktureller Hinsicht als auch ausgehend von ihrer inhaltlichen Gestaltung auf Interaktionen einwirken. Dabei variieren das Ausmaß ihrer Instrumentalisierung ebenso wie der daraus resultierende Einfluss auf die Standardisierung des Gesprächs. Zudem können sie nicht nur zum Gelingen von Kommunikation beitragen - wie im Fall ihres Einsatzes als Mittel, sich die Kooperationsbereitschaft der Jugendlichen zu sichern. Sie können diese auch gefährden, wenn der Einbezug von Dokumenten dazu führt, dass situative Verständigungshinweise und unmittelbare Klärungsmöglichkeiten reduziert werden und die daraus 
resultierende Standardisierung des Gesprächs dazu führt, dass Adressat_innen keine Möglichkeit erhalten, eigene Interpretamente vorzubringen.

\subsubsection{Ermitteln}

So konnten wir beobachten, dass Gespräche, in denen der Einbezug der Akte von Seiten der Sozialarbeiter_innen vergleichsweise dominant ist - die Akten liegen auf dem Besprechungstisch, es wird während der Interaktion in ihnen geblättert, aus ihnen vorgelesen -, sich im Hinblick auf die Interaktionsstrategien durch eine starke Ähnlichkeit zu polizeilichen Ermittlungsgesprächen auszeichnen. In diesen Fällen bilden die Akteninhalte, vor allem polizeiliche Meldungen und Anklageschriften, nicht nur die gegenüber den Adressat_innen kommunizierten Gesprächsanlässe oder fungieren als Stichwortgeber, sondern werden vielmehr als aktive Gesprächsteilnehmer mit dem Ziel der Sachverhaltserforschung in die Interaktionen einbezogen: Die Jugendlichen werden mit dem Inhalt der Anklageschrift konfrontiert und aufgefordert, sich dazu zu positionieren anstatt das Geschehen in ihren eigenen Worten wiederzugeben Dies illustriert der folgende Gesprächsauszug (Prot. 66, 5-6):

Frau Dreher: Da sollt ihr den [Vor- und Nachname eines Jungen] bedroht haben. Ich les' mal vor, was euch vorgeworfen wird. [liest aus der Anklageschrift vor: danach soll der Adressat mit zwei anderen 14-Jährigen einen Jungen, der an der Bushaltestelle wartete, in den Eingang eines Fachgeschäftes gedrängt und von ihm gefordert haben, ihnen sein Geld zu geben. Daraufhin habe der Junge ihnen sein leeres Portemonnaie gezeigt und später, wohl aus Angst, auch die leeren - Hosentaschen. Der Junge habe begonnen zu weinen]. Stimmt das so? Adressat: Ja.

Werden auf diese Weise die Möglichkeiten für die Adressat_innen, eigene Situationsdefinitionen zur Geltung zu bringen, in der Regel deutlich reduziert, kann der Einsatz von Informationen aus der Akte in der Interaktion überdies den Charakter der aus der richterlichen oder polizeilichen Praxis entlehnten Gesprächstechnik des Vorhalts annehmen. Dies geschieht, wenn Sozialarbeiter_innen, wie in dem nachfolgenden Beispiel (Prot. 46, 117-130), das Tatgeschehen entlang ihnen vorliegender Dokumente erheben. Die Sozialarbeiterin liest dem Adressaten in diesem Gespräch den strafrechtlichen Vorwurf direkt aus der Anklageschrift vor:

Frau Dreher: Was Ihnen vorgeworfen wird [beginnt aus der Anklageschrift vorzulesen: In der Tatnacht habe der Adressat in einem Getränkeladen mit einem Stein ein Fenster eingeworfen, mit dem Fuß oder Bein das Loch vergrößert, sich dabei verletzt und dann einen Kasten Bier entwendet. Er habe ein Klappmesser dabei gehabt].

Adressat: Dazu kann ich nur sagen, ich habe die Scheibe nicht eingeschlagen, die war bereits kaputt und ich hab' da ein wenig nachgeholfen. Ich war sehr betrunken, ich hatte den Tag noch nichts gegessen, in der Akte steht 1,8 Promille, das ist schon 'ne ganze Menge bei leerem Magen. Ich hab' dagegen getreten, weil ich was trinken wollte. Ich war alleine dabei, die anderen haben sich dann 
auch gewundert, dass ich nicht wiedergekommen bin, aber da war ich dann schon in der PG-Zelle [Polizeigewahrsam].

Frau Dreher: Sie wollten also noch was trinken.

Adressat: Ja.

Frau Dreher: Haben Sie zu der Zeit auch gekifft?

Adressat: Ja. Ich hab' auch viel getrunken. 1,8 sind viel.

Frau Dreher [liest weiter aus der Anklageschrift vor: Der Adressat habe sich auf der Flucht vor der Polizei unter einem Auto versteckt, von dort hätten ihn zwei Polizisten herausgezogen, der Adressat habe die Polizisten angespuckt, versucht zu beißen, im Streifenwagen habe er sie weiter beleidigt, z. B. ,ihr Wichser", ,ich bin euer Führer"]

Adressat: Das ist Quatsch, totaler Quatsch. Ich war so besoffen. In der Straße, wo das gewesen ist, da hab' ich den Kasten weggeschmissen und wollte unter das Auto, aber ich hab' da gar nicht drunter gepasst. Und da haben die Polizisten mich raus gezerrt und haben mir dann Handschellen angetan, das hat ziemlich weh getan. Ich hab' ihnen gesagt, wenn sie mir weh tun wollen, sollen mich schlagen. Ich war frech, aber ich hab' sie nicht beleidigt. Die haben mir auch den Kopf auf den Boden gedrückt, ich hab' davon auch Fotos gemacht, ich hatte hier [vermutlich deutet er auf sein Gesicht] überall blaue Flecken und Schrammen davon. Ich hab' gelacht und dann haben sie mich auch geschlagen. Ich hab' einen Schuh verloren, der lag am Tatort. An die Fahrt kann ich mich nicht erinnern, erst als wir im Präsidium waren und dann wollte ich nur schlafen. Am nächsten Tag wurde ich gefragt, ob ich eine Aussage machen will und dann haben die mich entlassen. Ich hatte aber nur noch einen Schuh. Ich bin dann mit einem Schuh nach Hause gegangen.

Frau Dreher: Hier steht, Sie haben versucht die Polizisten anzuspucken.

Adressat: Nein, das stimmt nicht. Auch nicht mal, als die mir die Handschellen angelegt haben. Ich hab' zu denen gesagt, die sollen nicht wie Mädchen schlagen. Und das hat die sauer gemacht. Die haben auch geschlagen.

Frau Dreher: Was ist mit diesem Ausdruck ,,ich bin euer Führer“?

Adressat: Nein. Ich hab' das gelesen und musste lachen.

Frau Dreher: Was war mit dem Klappmesser?

Adressat: Kann mich nicht daran erinnern. Ich meine, ich hatte keins dabei, vielleicht von 'nem Kollegen, das war auch nicht meine Hose und nicht meine Schuhe. Ich hab' mal ein Klappmesser gehabt, vor drei Jahren.

Die Sozialarbeiterin lässt den Adressaten völlig im Unklaren darüber, ob sie seinen der Aktenlage widersprechenden Äußerungen Glauben schenkt und enthält sich jeglicher Bewertung. Das Gespräch bekommt nicht nur eine starke Ähnlichkeit mit einem Verhör, wenn die Akte auf diese Weise als Informationsquelle genutzt wird. Im Kontext einer als ,diskursive Diagnostik“ (Scheffer 2001, S. $141 \mathrm{f}$.) zu charakterisierenden Gesprächsführung konkretisiert sich die Funktion von Akten als Informationsquelle für die Sozialarbeiter_innen mitunter auch in ihrer Instrumentalisierung als Gegenbeweis. So werden Informationen aus der Akte auch dazu herangezogen, um Aussagen der Adressat_innen zu widerlegen, d.h. die Akte ist 
nicht nur „Mitwisser“, sondern wird in der Interaktion zum „Besserwisser“ (Prot. 65, 67-70):

Frau Dreher: Haben Sie schon mal mit Polizei oder Gericht zu tun gehabt?

Adressat: Nein.

Frau Dreher: Doch, Sie sind einmal schwarzgefahren 2013.

Adressat: Ach so.

Frau Dreher: Das wurde eingestellt, war wohl nicht so dramatisch. Was war los am [Datum]?

Während im Fall dieser Interaktion der Widerspruch zwischen Akte und Aussage durch die Sozialarbeiterin mit Bezug auf die Einstellung des Verfahrens relativiert wird, kann es auch zu weiteren zahlreichen Nachfragen kommen, um mit Hilfe der Aktendokumentation den Wahrheitsgehalt von Aussagen und die Glaubwürdigkeit der Adressat_innen zu überprüfen. Der nachfolgende Protokollauszug (Prot. 44, 113-162) dokumentiert dieses Nachforschen am Detail besonders eindrücklich, wobei hier durch den Sozialarbeiter regelmäßig eine Anpassung der Aussagen des Adressaten an die Aktenlage vorgenommen wird.

Herr Arslan [hat die Akte auf dem Schoß]: Dann hab' ich diese Sache. Hier steht, dass du den [Name des Geschädigten] geschlagen haben sollst. Deswegen hat er auch bei der Polizei ausgesagt. [Beginnt aus der Akte vorzulesen:] Der [Name des Geschädigten] sagt, dass [Name des Adressaten] in der großen Pause Kaugummi in seine Haare gespuckt hat. Er hat das Kaugummi aus den Haaren entfernt und begab sich zum Cleanser [alle kichern], zum Mülleimer, um dieses dort zu entsorgen. Daraufhin habe [Name des Adressaten] versucht, den [Name des Geschädigten] am Hals zu verletzen. Erinnerst du dich daran? Adressat: Ja, aber nicht so. (113-114)

Statt dem Jugendlichen an dieser Stelle die Möglichkeit zu geben, die aus seiner Sicht von der Aktenlage abweichende Situation zu beschreiben, fährt der Sozialarbeiter fort, weitere Details zum Tathergang vorzutragen:

Herr Arslan: Von Mitschülern wurde dann erfahren, dass er von dir geschlagen wurde. Die haben ihn dann in den Sanitätsraum getragen. Dann hat er hier ein Attest [liest es vor], dass ihm bescheinigt, wo er dann eine Gehirnerschütterung hatte [blättert weiter in der Akte]. Das ist attestiert worden. Keine Teilnahme an sportlichen Tätigkeiten für vier Wochen, zwei Wochen keine Spielgeräte.

Mutter [leise]: Also keine Playstation.

Herr Arslan: War das so, wie er das beschrieben hat?

Adressat: Das mit dem Kaugummi war ich nicht, das war einer neben mir. Und er hat es dann auf mich geworfen. Ich wollte es dann wegwerfen und auf dem Weg zum Mülleimer ist er von hinten auf mich rauf. Hat mich gewürgt. Ich hab' ihn dann weggeschubst und dann ist er wieder auf mich rauf und ich hab' ihn wieder weggeschubst. Dann kam er nochmal und ja, dann habe ich ihn geschlagen.

Herr Arslan: Also hast du beim dritten Mal zugeschlagen.

Adressat: Ja, weil, ich wusste mir nicht anders zu helfen. (115-120) 
Das an dieser Stelle des Gesprächs vom Adressaten formulierte Schuldeingeständnis, den Jungen geschlagen zu haben, übergeht der Sozialarbeiter. Auch das von ihm vorgebrachte entschuldigende Interpretament, aus Notwehr gehandelt zu haben, findet keine interaktive Anerkennung. Stattdessen fokussiert Herr Arslan nicht nur weitere Details der Situation, sondern führt, im wechselnden Modus von Frage und Vorhalt, einen Abgleich der Aussage des Adressaten mit den Informationen aus der Akte durch:

Herr Arslan: Wie hast du ihn geschlagen?

Adressat: Ich wollte es nicht. Hab' ihn hier im Gesicht getroffen.

Herr Arslan: Und dann ist er gegen den Schrank mit dem Hinterkopf geschlagen?

Adressat: Ja, ein bisschen.

Herr Arslan: Ist er hingefallen?

Adressat: Nein, zwei Mitschüler haben ihn festgehalten.

Herr Arslan: Aber er war taumelig. Er sagt, zwei Mitschüler haben ihn in den Sanitätsraum getragen.

Adressat: Nein, er konnte gehen.

Herr Arslan: Du sagst, er hat dich von hinten gewürgt, nachdem er Kaugummi auf dich gespuckt hat.

Adressat: Ja.

Herr Arslan: Wie kommt es, dass er Kaugummi auf dich spuckt? (121-131)

An dieser Stelle des Gespräches bricht der Sozialarbeiter den Versuch, Aussage und Aktenlage in Übereinstimmung zu bringen, unvermittelt ab. Stattdessen wendet er sich mit der Frage nach dem Grund des Kaugummi-Spuckens den Ursachen des Konflikts zu und fragt auch danach, ob die Jugendlichen anschließend weiterhin in Streitigkeiten geraten seien. In vergleichbar unvermittelter Weise wechselt er daraufhin erneut das Thema und bringt mit der Frage nach weiteren Zeugen des Vorfalls seine Zweifel an der Glaubwürdigkeit des Adressaten zum Ausdruck:

Herr Arslan: Du hast dich entschuldigt?

Adressat: Ja.

Herr Arslan: Schriftlich oder mündlich?

Adressat: Mündlich.

Herr Arslan: Was hast du dazu gesagt?

Adressat: [Name des Geschädigten], tut mir leid, was passiert ist.

Herr Arslan: Weißt du, wer ihn ins Sanitätszimmer getragen hat?

Adressat: [nennt zwei Namen von Mitschülern, jeweils Vor- und Nachnamen] Herr Arslan [wiederholt die Namen, hat Schwierigkeiten mit der Aussprache und lässt sich die Namen buchstabieren, notiert sie sich]: O.k. und die Lehrerin? Adressat: Ich glaub', es war zum Zeitpunkt kein Lehrer dabei.

Herr Arslan: Haben die beiden was erzählt über die Verletzung?

Adressat: Nein, keiner von beiden.

Herr Arslan: Du hast bei der Polizei ausgesagt. [Liest die polizeiliche Zusammenfassung der Aussage des Adressaten vor: es ist normal, dass sich Schüler mit Kaugummi bewerfen, der Geschädigte hat den Adressaten gewürgt, woraufhin dieser den Geschädigten geschlagen habe.] Das hast du auch gesagt? 
Adressat: Ja.

Herr Arslan: Wo hast du ihn getroffen?

Adressat: Ich hab' ihn im Bereich Kinn, Mund getroffen.

Herr Arslan: [Name des Geschädigten] ist dann von zwei Mitschülern aus dem

Zimmer gebracht worden. Hat die Polizei die beiden auch befragt?

Adressat: Glaube nicht. (145-162)

Insbesondere in der Instrumentalisierung der Akte als Beweis offenbart sich der „performative Charakter“ (vgl. Muckel 1997, S. 132 ff.) von Dokumenten, d.h. die Wirkmacht des in den Dokumenten Repräsentierten, in der und auf die Interaktion zwischen Sozialarbeiter_innen und ihren Adressat_innen. Die Akte ist hier nicht nur Instrument für die Verwaltung, sondern wird überdies zum „Maßstab für die Gestaltung der ,ersten Wirklichkeit““ (ebd., S. 133). Unsere Beobachtungen deuten darauf hin, dass es sich bei diesem detailgenauen Ermitteln, wie es Herr Arslan in obigem Beispiel betreibt, um eine Form der Diagnostik handelt, die vor allem nicht anwesende Beteiligte am Prozess überzeugen soll. Sie zielt darauf, Akte und Aussage in Kongruenz zu bringen, um ein formal korrektes Abfassen des Berichts - der diese Interaktionen ebenfalls sowohl in struktureller als auch in inhaltlicher Hinsicht prägt - und die Auswahl der passenden Maßnahme zu ermöglichen.

Dass das damit unweigerlich verbundene Risiko, die Kooperationsbereitschaft der Adressat_innen zu gefährden, freiwillig von den Fachkräften in Kauf genommen wird, zeigt sich daran, dass sich in unserem Datenmaterial auch Gesprächsauszüge identifizieren lassen, in denen sich die Sozialarbeiter_innen in der Interaktion mit den Adressat_innen nicht in den zuvor beschriebenen Weisen auf Dokumente stützten. Hierfür stehen Sequenzen, in denen sie die Angaben der Adressat_innen nicht mit Informationen aus der Akte abglichen, sondern stattdessen deren Glaubwürdigkeit betonen, wie folgende (Prot. 11, 76), in der eine Sozialarbeiterin ihrem Adressaten versichert:

Frau Sommer: Du kannst mir erzählen, was du willst - ich glaube dir, warum soll ich dir nicht glauben.

Eine positive Bescheinigung der Glaubwürdigkeit des Adressaten erfolgt auch in einem anderen Gespräch derselben Sozialarbeiterin (Prot. 05, 73), in dem sie zudem verdeutlicht, dass für sie nicht die Aktenlage entscheidend ist, weil sie sich stattdessen auf ihr Bauchgefühl verlässt:

Frau Sommer: Mein Bauch sagt mir, ich glaube Ihnen.

Ohne Einbezug von Dokumenten vergrößert sich der Möglichkeitsspielraum der Adressat_innen, ihre Sichtweise im Gespräch in ihren eigenen Worten darzulegen, wodurch die interaktive Asymmetrie zwischen Sozialarbeiter_innen und Adressat_innen reduziert wird. Dies illustrieren die folgenden Sequenzen (Prot. 35, 5-10), welche den Beginn eines Gesprächs mit einem Jugendlichen wiedergeben, der mit Cannabis erwischt wurde:

Frau Sommer: Nimm Platz. So, ich hab' dich eingeladen. Warum bist du heute hier? 
Adressat: Weil ich Marihuana gekauft hab' und erwischt wurde.

Frau Sommer: Wie kommt das?

Adressat: Durch Freunde. In meinem Freundeskreis wird das viel gemacht und wollte das auch ausprobieren.

Frau Sommer: Das heißt, was für eine Situation war das?

Adressat: Das war sehr unangenehm für mich.

Anders als im obigen Beispiel (vgl. Prot. 66) werden dem Adressaten nicht Auszüge aus der Anklageschrift vorgelesen und er wird nicht gefragt „stimmt das so?“, sondern die Sozialarbeiterin fragt ganz offen nach dem Anlass des Termins und stellt Nachfragen.

Offene Einstiegsfragen ohne den Bezug auf Informationen aus der Akte (z.B. Warum bist du heute hier?) und erzählgenerierende Nachfragen (z. B. Wie kommt das?, Was für eine Situation war das?, Erzähl mal, was war da los?) erwecken den Eindruck, dass Sozialarbeiter_innen unvoreingenommen sind und für sie die Sichtweise der Adressat_innen Relevanz besitzt.

An diesen Beispielen für den Verzicht auf den Einbezug von Dokumenten zeigen sich die Gesprächsgestaltungsmöglichkeiten von Sozialarbeiter_innen, mithin Begrenzungen der Wirkmächtigkeit der Dokumente auf die Gestaltung der Interaktion mit den Adressat_innen. Sie illustrieren folglich, dass Fachkräfte das Risiko, die Kooperationsbereitschaft der Adressat_innen zu gefährden - etwa durch die Inanspruchnahme von Dokumenten zum Zweck der Sachverhaltserforschung, die mitunter die Form des Vorhalts oder des Gegenbeweises annehmen kann - ohne äußeren Zwang eingehen.

Auf welche Veränderungen die von uns identifizierten Funktionen von Dokumenten in der Interaktion in Bezug auf die sozialarbeiterische Handlungspraxis und das von Peters und Cremer-Schäfer postulierte Helferselbstverständnis hindeuten, diskutieren wir abschließend vor dem Spiegel der Originalstudie.

\section{Kontinuitäten und Diskontinuitäten im zeitlichen Vergleich}

Betrachtet man die Ergebnisse unserer Analyse vor dem Hintergrund der Originalstudie, so stehen sie teilweise mit den darin postulierten interaktionistischen Annahmen im Einklang. Wie eingangs erwähnt, gingen Peters und Cremer-Schäfer (1975, S. 16ff.) davon aus, dass das Handeln der Sozialarbeiter_innen davon geleitet war, ihr Selbstverständnis als Helfer_in aufrechtzuerhalten, Konflikte zu vermeiden und die Hilfeabsicht die Interaktion bestimmen zu lassen. Zudem stellten sie fest, dass die von ihnen beobachteten Sozialarbeiter_innen kein originäres Interesse an der Feststellung von Schuld hatten, sondern stets versuchten, die Realität so auszuhandeln und die Adressat_innen in einer Weise zu typisieren, dass stigmatisierende Etikettierungen erschwert werden, z.B. indem sie die Adressat_innen als Ersttäter_innen mit geregeltem Arbeitsverhältnis darstellten (vgl. ebd., S. 32f.). Situationsdefinitionen der Adressat_innen (z.B. Unschuldsbeteuerungen) wurden von den Sozialarbeiter_innen entweder vorbehaltlos anerkannt oder ihnen wurde zumindest nicht widersprochen. Mitunter unterstützten die Sozialarbeiter_innen die Situations- 
definitionen der Adressat_innen sogar im Hinblick auf ihre Durchsetzungschancen vor Gericht, z. B. durch Hinweise, etwa auf die Rolle von Zeugen (vgl. ebd., S. 33). Auf diese Weise lassen sich auch einige der von uns identifizierten Funktionen von Dokumenten in der Interaktion interpretieren. Der Einsatz der Akte, um gesichtsbedrohende Themen zu neutralisieren und Rollenkonflikte zu vermeiden, kann ebenso als Versuch verstanden werden, die Interaktion so zu beeinflussen, dass Konflikte vermieden werden und die Hilfeabsicht die Interaktion bestimmt.

In unserer Analyse deuten sich jedoch auch Abweichungen von den Annahmen und Befunden der Originalstudie an. Der Einsatz von Dokumenten, um zu ermitteln und die Glaubwürdigkeit der Adressat_innen zu prüfen, steht im Widerspruch dazu, stigmatisierende Etikettierungen zu erschweren oder Situationsdefinitionen der Adressat_innen zu unterstützen. Diese Funktionen der Akte scheinen vielmehr in Strategien der Wahrheitsfindung eingebettet zu sein, wobei nicht die Hilfeabsicht für die Adressat_innen, sondern für die Justiz im Vordergrund steht. Liegt das Ziel darin, einen möglichst korrekten Bericht für das Gericht zu verfassen und einen passenden Maßnahmenvorschlag zu eruieren, deuten dann auch Selbstpositionierungen von befragten Sozialarbeiter_innen darauf hin, dass der Einbezug von Dokumenten in das Gespräch in erster Linie am Gericht und der Staatsanwaltschaft als den eigentlichen Rezipienten des JGH-Berichts orientiert ist. Ihnen gegenüber soll - in Antizipation juristischer Kriterien wie etwa Korrektheit und Distanziertheit (zur Definition der juristischen Berufsrolle vgl. Lautmann 1972) - die eigene Kompetenz und Professionalität unter Beweis gestellt werden. ${ }^{15}$

Der Einsatz von Dokumenten in der Interaktion zu diesen Zwecken untermauert den schon an anderer Stelle (vgl. Kühne et al. 2017) konstatierten Wandel des von Peters und Cremer-Schäfer postulierten Helferselbstverständnisses. In der Originalstudie kam im Sprechen über den Bericht eine Selbstpositionierung zum Ausdruck, die sich klar an der Hilfeabsicht für die Adressat_innen orientiert. Dies illustriert eine hierfür typische Sequenz aus einem Protokoll von Peters und Cremer-Schäfer (Prot. 66 JGH B, 11), in der ein Sozialarbeiter dem Adressaten seine Rolle erläutert:

Sozialarbeiter: Wir hier, das Jugendamt, sind Vertreter Ihrer Interessen. Ich habe deswegen einige Fragen zu Ihrem Lebenslauf.

Auch in den folgenden Sequenzen (Prot. 56 JGH B, 49-53) eines Gesprächs zwischen einem Sozialarbeiter, dem Vater und der Großmutter eines Adressaten, der angeklagt ist, weil er in betrunkenem Zustand einen Verkehrsunfall verursacht haben soll, zeigt sich deutlich eine Parteilichkeit für den Adressaten:

Sozialarbeiter: Den Führerschein wird er los.

Vater: Ach, noch länger?

Sozialarbeiter: Ja, ich glaube das. Ich habe nicht darüber zu entscheiden, aber ich glaube das.

Großmutter: Das ist aber sehr schlecht. Er fährt mich doch immer. Ich kann doch so schlecht mit dem Zug oder der Straßenbahn fahren.

$15 \mathrm{Zu}$ den Darstellungsleistungen in JGH-Berichten vgl. Gohde und Wolff (1990). 
Sozialarbeiter: Ach so. Ja, das werde ich dann auch noch vortragen. [Der Sozialarbeiter notiert, leise vor sich hinsprechend:] Großmutter hat eine Armverletzung und eine noch nicht verheilte Verletzung am Bein. [Spricht lauter:] Ja, ich werde das sagen, vielleicht hole ich damit zwei Monate heraus.

Der Sozialarbeiter lässt offenbar werden, dass er mit seinen Berichtsinformationen wie ein Strafverteidiger im Sinne seines Mandanten vor Gericht taktieren wird und nutzt hierfür einen anwaltsähnlichen Sprachgebrauch.

In unserem Datenmaterial dagegen kommt in der Bezugnahme auf den Bericht in den Gesprächen eine Selbstpositionierung von Sozialarbeiter_innen zum Ausdruck, wonach sie sich primär als neutrale Berichterstatter_innen verstehen. Dies demonstriert der folgende Gesprächsauszug (Prot. 44, 10-12), in dem der Sozialarbeiter die Mutter seines Adressaten fragt:

Herr Arslan: Sie wissen, was meine Aufgabe ist?

Mutter: Nicht so richtig.

Herr Arslan: Ich schreibe einen Bericht für die Staatsanwaltschaft oder eben den Richter. Was Sie mir erzählen, werde ich aufschreiben und so weitergeben. Wenn ich vor Gericht gefragt werde, als Zeuge muss ich sagen, was Sie mir gesagt haben. Das ist meine Aufgabe.

Der Bezug auf Dokumente dient dem Sozialarbeiter hier als Mittel zur Selbstpositionierung als Helfer für das Gericht. Insofern zeigt der diachrone Vergleich, dass hinsichtlich des Verweises auf den Bericht in den Gesprächen zwischen Sozialarbeiter_innen und ihren Adressat_innen eine Verschiebung von Selbstpositionierungen in Richtung ihres justiziellen Hilfeauftrags stattgefunden hat.

\section{Fazit}

Unsere Ergebnisse deuten an, dass gerade der zurückhaltende Einsatz von Dokumenten in der Interaktion mit den Adressat_innen als Indiz für die Fortexistenz eines Helferselbstverständnisses im Sinne von Peters und Cremer-Schäfer gilt. Hierfür stehen exemplarisch die Gesprächsauszüge, in denen Sozialarbeiter_innen gar keine Selbstpositionierung vor- und auch nicht auf den Bericht Bezug nehmen, sondern ganz offen fragen „Warum bist du heute hier?“.

Zwar stellen Selbstpositionierungen nur eine Komponente des beruflichen Selbstverständnisses dar, das darüber hinaus ethische Orientierungen, Handlungsmaximen, Haltungen und Bewertungskonzepte umfasst (vgl. Lutz 2010, S. 95). Mit dem Ausbleiben einer Selbstpositionierung resp. der Selbstpositionierung als Berichterstatter_in korrespondieren jedoch auch spezifische Ansichten über den Bericht, was sich in unseren Interviews mit den Sozialarbeiter_innen gezeigt hat: Neutrale Berichterstatter_innen sehen den Bericht als Hilfe für das Gericht und filtern die Informationen für ihren Bericht nicht. Sie plädieren für Vollständigkeit und führen auch Aspekte, die sich für die Adressat_innen nachteilig auswirken können, wie z.B. Vorbelastungen, in ihrem Bericht auf. Sozialarbeiter_innen, die sich nicht über die Berichtspflicht definieren und ihre Gespräche nicht strikt nach dem Bericht struk- 
turieren, betrachten den Bericht dagegen als taktisches Instrument. Sie überlegen genau, was sie in den Bericht schreiben und selektieren Informationen zum Vorteil der Adressat_innen, um im Gerichtssaal „für Stimmung zu sorgen“, wie es eine Sozialarbeiterin (Frau Lindner) ausgedrückt hat. Umgekehrt lassen sie Informationen (z.B. Vorbelastungen) weg, die sich nachteilig für ihre Adressat_innen auswirken können. ${ }^{16}$

Ihre Gespräche zeichnen sich zudem durch einen diskreteren Gebrauch der Akte aus. Wie schon erwähnt, liegt diese zuweilen nicht auf dem Besprechungstisch, sondern auf dem Schreibtisch oder bleibt in der Tasche. Damit vermitteln die Sozialarbeiter_innen den Eindruck von Unvoreingenommenheit und, dass die Sichtweise der Adressat_innen für sie im Vordergrund steht. Allerdings fungieren Akten für sie ebenfalls als objektive Informanten, d.h. sie werden auch von ihnen zu Hilfe genommen, wenn Widersprüche auftreten oder wenn das Gespräch stockt, z.B. weil die Adressat_innen nicht ausreichend auskunftsfreudig oder erzählkompetent sind, um den Anlass des Gesprächs in ihren eigenen Worten wiederzugeben. Auch wird der Wahrheitsgehalt der Akteninformationen von ihnen nicht in Frage gestellt: Polizeiberichte und Anklageschriften werden vielmehr behandelt, als wären sie objektive Dokumentationen von Tatbeständen oder „Abbildungen von Wirklichkeit“ (Lau und Wolff 1981, S. 201; vgl. auch Sagebiel 1974). D. h. auch der zurückhaltende Einsatz von Dokumenten bedeutet keine uneingeschränkte Parteinahme für die Adressat_innen und auch nicht, dass der justizielle Hilfeauftrag vernachlässigt oder weniger korrekt erfüllt wird. Ebenso erweist sich der Umkehrschluss, dass Sozialarbeiter_innen, die sich als neutrale Berichterstatter_innen verstehen, keine Hilfe für die Adressat_innen leisten, als unzulässig. Auch wenn die Berichtspflicht die Gespräche von z. B. Herrn Matthäus dominiert, schlägt sich seine Reduktion auf die Berichterstatterrolle nicht in der Weise in seiner Handlungspraxis nieder, dass er keine Hilfeleistungen für seine Adressat_innen erbringt. Vielmehr ist das Ausmaß an Unterstützung, das er den Adressat_innen gewährt, indem er sie über den Ablauf der Gerichtsverhandlung informiert oder ihnen Ratschläge gibt, wie sie deren Ausgang positiv beeinflussen können, davon abhängig, ob sich die Adressat_innen an der Aufrechterhaltung einer angenehmen Gesprächsatmosphäre beteiligen. Angesichts dessen, dass es demzufolge auch auf die Performance der Adressat_innen in der Interaktion ankommt, können situative Faktoren gleichermaßen Einfluss auf die Handlungspraxis ausüben.

Dennoch zeigt unsere Analyse, dass die Sequenz „Meine Aufgabe ist, einen Bericht zu schreiben“ die Struktur des Gesprächs sowie den Einbezug von Dokumenten in die Interaktion bestimmen und gleichzeitig Ausdruck der Selbstpositionierung der Sozialarbeiter_innen sein kann. Unsere Ergebnisse bekräftigen die These, dass der Einsatz von Dokumenten in der Interaktion mit den Adressat_innen die Gefahr in sich birgt, dass Sozialarbeiter_innen ihre Handlungspraxis standardisieren und dabei eine juristische Handlungslogik antizipieren. Denn charakterisierten Lau und Wolff (1981, S. 199) das Verhältnis von Sozialarbeiter_innen zu ihren Akten Anfang der 1980er Jahre noch als ein „Bündnis wider Willen“, deuten unsere Befunde nicht auf

${ }^{16}$ Eine Sozialarbeiterin (Frau Lindner) argumentiert, dass Richter im Bundeszentralregister nachsehen können, wenn sie sich dafür interessieren. 
ein „diffuse[s] Unbehagen mit der Akte“ (ebd., S. 207), sondern, angesichts der sich insbesondere im Modus des Ermittelns offenbarenden (Zuschreibungs-)Kooperation, vielmehr auf Tendenzen einer Allianz mit der Akte hin. In variierendem Ausmaß fungiert sie als Verbündete in der Interaktion und erweitert das sozialarbeiterische Handlungsrepertoire um Möglichkeiten, sich als Helfer_in darzustellen - jedoch nicht nur der Adressat_innen, sondern vor allem des Gerichts.

Funding Open Access funding provided by Projekt DEAL.

Open Access Dieser Artikel wird unter der Creative Commons Namensnennung 4.0 International Lizenz veröffentlicht, welche die Nutzung, Vervielfältigung, Bearbeitung, Verbreitung und Wiedergabe in jeglichem Medium und Format erlaubt, sofern Sie den/die ursprünglichen Autor(en) und die Quelle ordnungsgemäß nennen, einen Link zur Creative Commons Lizenz beifügen und angeben, ob Änderungen vorgenommen wurden.

Die in diesem Artikel enthaltenen Bilder und sonstiges Drittmaterial unterliegen ebenfalls der genannten Creative Commons Lizenz, sofern sich aus der Abbildungslegende nichts anderes ergibt. Sofern das betreffende Material nicht unter der genannten Creative Commons Lizenz steht und die betreffende Handlung nicht nach gesetzlichen Vorschriften erlaubt ist, ist für die oben aufgeführten Weiterverwendungen des Materials die Einwilligung des jeweiligen Rechteinhabers einzuholen.

Weitere Details zur Lizenz entnehmen Sie bitte der Lizenzinformation auf http://creativecommons.org/ licenses/by/4.0/deed.de.

\section{Literatur}

Böhringer, Daniela. 2015. Formulare in Aktion - Zur Herstellung von Dokumenten in der Arbeitsverwaltung. In Mediatisierung (in) der Sozialen Arbeit, Hrsg. Nadia Kutscher, Thomas Ley, und Udo Seelmeyer, 260-277. Baltmannsweiler: Schneider Hohengehren.

Braun-Thürmann, Holger. 2006. Ethnografische Perspektiven: Technische Artefakte in ihrer symbolischkommunikativen und praktisch-materiellen Dimension. In Technografie. Zur Mikrosoziologie der Technik, Hrsg. Werner Rammert, Cornelius Schubert, 199-221. Frankfurt a.M., New York: Campus.

Cicourel, Aaron. 1968. The social Organisation of juvenile justice. New York: Wiley.

Cremer-Schäfer, Helga. 2012. Kritische Institutionenforschung. Eine Forschungstradition, an der weiter gearbeitet werden kann? In Kritisches Forschen in der Sozialen Arbeit, Hrsg. Elke Schimpf, Johannes Stehr, 135-148. Wiesbaden: VS.

Cremer-Schäfer, Helga. 2018. Die sanften Kontrolleure - 1975 und im Alter von über vierzig. Helga Cremer-Schäfer im Gespräch mit Rebekka Streck zu einer fast vergessenen ethnografischen Studie. In Doing Social Work - Ethnografische Forschung als Theoriebildung, Hrsg. Kathrin Aghamiri, et al., 21-37. Opladen: Barbara Budrich.

Deppermann, Arnulf. 2008. Gespräche analysieren. Eine Einführung. Wiesbaden: Springer VS.

Garfinkel, Harold. 1956. Conditions of successful degradation ceremonies. American Journal of Sociology 61(5):420-424.

Garfinkel, Harold. 1967. Studies in ethnomethodology. Englewood Cliffs: Prentice Hall.

Garfinkel, Harold. 1974. The origins of the term „ethnomethodology“. In Ethnomethodology: selected readings, Hrsg. Roy Turner, 15-18. Harmondsworth: Penguin.

Goffman, Erving. 1974. Das Individuum im öffentlichen Austausch. Mikrostudien zur öffentlichen Ordnung. Frankfurt a.M.: Suhrkamp.

Gohde, Hellmuth, und Stephan Wolff. 1990. „Gutachterlichkeit“ in der Jugendgerichtshilfe. Neue Praxis 20:316-328.

Haferkamp, Hans, und Günter Meier. 1972. Sozialarbeit als Instanz sozialer Kontrolle. Kriminologisches Journal 4(2):100-115.

Hitzler, Sarah, und Heinz Meßmer. 2008. Gespräche als Forschungsgegenstand in der Sozialen Arbeit. Zeitschrift für Pädagogik 54(2):244-260.

Hydén, Lars-Christer. 1994. The Social Worker as Moral Worker. Applying for Money - the Moral Encounter between Social Workers and Clients. In Text and Talk in Professional Context, Hrsg. BrittLouise Gunnarsson, Per Linell, und Bengt Nordberg, 187-199. Uppsala: ASLA. 
Kessl, Fabian. 2013. Soziale Arbeit in der Transformation des Sozialen. Eine Ortsbestimmung. Wiesbaden: Springer VS.

Kessl, Fabian, und Hans-Uwe Otto. 2009. Soziale Arbeit ohne Wohlfahrtsstaat? In Soziale Arbeit ohne Wohlfahrtsstaat? Zeitdiagnosen, Problematisierungen und Perspektiven, Hrsg. Fabian Kessl, HansUwe Otto, 7-21. Weinheim: Beltz.

Knoblauch, Hubert, und Christian Heath. 1999. Technologie, Interaktion und Organisation: die Workplace Studies. Schweizerische Zeitschrift für Soziologie 25(2):163-181.

Kühne, Sylvia, Christina Schlepper, und Jan Wehrheim. 2017. „Die sanften Kontrolleure“ (Helge Peters und Helga Cremer-Schäfer 1975) revisited. Soziale Passagen 9(2):329-344.

Kutscher, Nadia. 2018. Soziale Arbeit und Digitalisierung. In Handbuch Soziale Arbeit, Hrsg. Hans-Uwe Otto, Hans Thiersch, Rainer Treptow, und Holger Ziegler, 1430-1440. München: Reinhardt.

Kutscher, Nadia, Thomas Ley, und Udo Seelmeyer. 2015. Mediatisierung (in) der Sozialen Arbeit. Baltmannsweiler: Schneider Hohengehren.

Latour, Bruno. 1995. Wir sind nie modern gewesen. Versuch einer symmetrischen Anthropologie. Berlin:: Akademie-Verlag.

Lau, Thomas, und Stephan Wolff. 1981. Bündnis wider Willen - Sozialarbeiter und ihre Akten. Neue Praxis 11(3):199-214.

Lautmann, Rüdiger. 1972. Justiz - Die stille Gewalt. Frankfurt a.M.: Athenäum.

Ley, Thomas. 2010. „Unser Schreibzeug arbeitet mit an unseren Gedanken.“ Oder: Zur Konstruktion des sozialpädagogischen Falles in computerisierten Arbeitsumgebungen. In Soziale Arbeit und Medien, Hrsg. Georg Cleppien, Ulrike Lerche, 219-233. Wiesbaden: VS.

Ley, Thomas, und Udo Seelmeyer. 2014. Dokumentation zwischen Legitimation, Steuerung und professioneller Selbstvergewisserung. Sozial Extra 38(4):51-55.

Lutz, Tilman. 2010. Soziale Arbeit im Kontrolldiskurs. Jugendhilfe und ihre Akteure in postwohlfahrtstaatlichen Gesellschaften. Wiesbaden: VS.

Merchel, Joachim. 2004. Pädagogische Dokumentation zwischen Etikettierung und Ausweis fachlichen Handelns. In Dokumentation pädagogischer Arbeit. Grundlagen und Methoden für die Praxis der Erziehungshilfen, Hrsg. Heinz Henes, Wolfgang Trede, 15-41. Frankfurt a.M.: IGfH.

Merchel, Joachim, und Wolfgang Tenhaken. 2015. Dokumentation pädagogischer Prozesse in der Sozialen Arbeit: Nutzen durch digitalisierte Verfahren. In Mediatisierung (in) der Sozialen Arbeit, Hrsg. Nadia Kutscher, Thomas Ley, und Udo Seelmeyer, 171-191. Baltmannsweiler: Schneider Hohengehren.

Muckel, Petra. 1997. Der Alltag mit Akten - psychologische Rekonstruktion bürokratischer Phänomene: Eine empirische Untersuchung in verschiedenen Institutionen auf der Grundlage der Grounded Theory. Aachen: Shaker.

Nadai, Eva. 2012. Von Fällen und Formularen: Ethnographie von Sozialarbeitspraxis im institutionellen Kontext. In Kritisches Forschen in der Sozialen Arbeit, Hrsg. Elke Schimpf, Johannes Stehr, 149-163. Wiesbaden: VS.

Nadai, Eva. 2015. „Bereitschaft zur Arbeitsaufnahme“. Zur Rolle von Dokumenten für die Verwaltung von Arbeitslosigkeit. In Mediatisierung (in) der Sozialen Arbeit, Hrsg. Nadia Kutscher, Thomas Ley, und Udo Seelmeyer, 242-259. Baltmannsweiler: Schneider Hohengehren.

Peters, Helge, und Helga Cremer-Schäfer. 1975. Die sanften Kontrolleure. Wie Sozialarbeiter mit Devianten umgehen. Stuttgart: Ferdinand Enke.

Prior, Lindsay. 2008a. Repositioning documents in social research. Sociology 42(5):821-836.

Prior, Lindsay. 2008b. Documents and action. In The Sage handbook of social research methods, Hrsg. Pertti Alasuutari, Leonhard Bickman, und Julia Brannen, 479-492. London: SAGE.

Sagebiel, Felizitas. 1974. Definitionspotential der Jugendgerichtshilfe beim Kriminalisierungsprozeß. Kriminologisches Journal 6(3):232-235.

Scheffer, Thomas. 2001. Asylgewährung. Eine ethnographische Analyse des deutschen Asylverfahrens. Stuttgart: Lucius \& Lucius.

Serres, Michel. 1987. Der Parasit. Frankfurt a.M.: Suhrkamp.

Smith, Dorothy E. 1974. The social construction of documentary reality. Social Inquiry 44(4):257-268.

Smith, Dorothy E. 2005. Institutional ethnography: a sociology for people. Lanham: AltyMira.

Stukenbrock, Anja. 2013. Sprachliche Interaktion. In Sprachwissenschaft: Grammatik - Interaktion - Kognition, Hrsg. Peter Auer, 217-260. Wiesbaden: Springer.

Taylor, Carolyn, und Susan White. 2000. Practicing reflexivity in health and welfare. Making knowledge. Maidenhead: Open University Press.

Wolff, Stephan. 2005. Dokumenten- und Aktenanalyse. In Qualitative Forschung. Ein Handbuch, Hrsg. Uwe Flick, Ernst von Kardorff, und Ines Steinke, 502-513. Reinbek: Rowohlt. 